\title{
Effect of Docosahexaenoic Acid on a Biomarker of Head Trauma in American Football.
}

Jonathan M. Oliver, $\mathrm{PhD}^{1}$; Margaret T. Jones, $\mathrm{PhD}^{2}$; K. Michele Kirk, $\mathrm{MD}^{1,3,4}$; David A. Gable, MSc ${ }^{1,3}$; Justin T. Repshas, $\mathrm{MSc}^{1}$; Torie A. Johnson, $\mathrm{MSc}^{1}$; Ulf Andréasson, $\mathrm{PhD}^{6}$; Niklas Norgren, $\mathrm{PhD}^{5}$; Kaj Blennow, $\mathrm{MD}, \mathrm{PhD}^{6}$; Henrik Zetterberg, MD, $\mathrm{PhD}^{6,7}$

${ }^{1}$ Sports Concussion Research Group, Department of Kinesiology, Texas Christian University, Fort Worth, TX

${ }^{2}$ Division of Health and Human Performance, George Mason University, Manassas, VA

${ }^{3}$ Department of Sport Medicine, Texas Christian University, Fort Worth, TX

${ }^{4}$ Department of Sport Medicine, John Peter Smith Hospital, Fort Worth, TX

${ }^{5}$ UmanDiagnostics, Umeå, Sweden

${ }^{6}$ Clinical Neurochemistry Laboratory, Institute Of Neuroscience and Physiology, the Sahlgrenska Academy at University of Gothenburg, Mölndal, Sweden

${ }^{7}$ Department of Molecular Neuroscience, UCL Institute of Neurology, Queen Square, London, UK

Corresponding Author:

Jonathan M. Oliver, PhD

Texas Christian University

Sports Concussion Research Group

Department of Kinesiology

TCU Box 297730

Fort Worth, TX 76129

(817)257-5623 Office

(817)257-7702 Fax

jonathan.oliver@tcu.edu 


\begin{abstract}
PURPOSE: American football athletes are exposed to subconcussive impacts over the course of the season resulting in elevations in serum neurofilament light (NFL), a biomarker of axonal injury. Docosahexaenoic acid (DHA) has been reported to reduce axonal trauma associated with traumatic brain injury in rodent models. However, the optimal dose in American football athletes is unknown. This study examined the effect of differing doses of DHA on serum NFL over the course of a season of American football.

METHODS: In a randomized, double-blind, placebo-controlled, parallel design, 81 National Collegiate Athletic Association Division I American football athletes were assigned to ingest either 2, 4, $6 \mathrm{~g} \cdot \mathrm{d}$ of DHA or placebo. Blood was sampled at specific times over the course of $189 \mathrm{~d}$, coincident with changes in intensity, hours of contact, and likely changes in head impacts. Standardized magnitude-based inference was used to define outcomes.

RESULTS: DHA supplementation increased plasma DHA in a dose-dependent manner $(2 \mathrm{~g} \cdot \mathrm{d}$ : mean difference from baseline; $\pm 90 \% \mathrm{CL} ; 2 \mathrm{~g} \cdot \mathrm{d}: 1.3 ; \pm 0.6 ; 4 \mathrm{~g} \cdot \mathrm{d}: 1.6 ; \pm 0.7 \% ; 6 \mathrm{~g} \cdot \mathrm{d}: 2.8 ; \pm 1.2 \%)$. Serum NFL increased to a greater extent in starters (area under the curve, $1995 \pm 1383 \mathrm{pg} \cdot \mathrm{mL}$ ) versus nonstarters (1398 $\pm 581 \mathrm{pg} \cdot \mathrm{mL} ; \mathrm{P}=0.024)$. Irrespective of dose, supplemental DHA likely attenuated serum NFL coincident with increases in serum NFL by likely small and moderate magnitude (effect size $=0.4-0.7$ ).

CONCLUSIONS: Findings from this study, the first large-scale study examining potential prophylactic use of DHA in American football athletes, include identification of optimal dose of DHA, suggesting a neuroprotective effect of DHA supplementation.
\end{abstract}




\section{Introduction}

American football is associated with the highest incidence of concussion (16), often used interchangeably with the term mild traumatic brain injury (mTBI), among team sports (19). This is not surprising, given that during play American football athletes are routinely exposed to head impacts, which result in head rotational acceleration/deceleration forces (inertial loading) and propagation of force through the brain $(9,10,14,15,17,40,42)$. These mechanical forces are known to result in stretching of white matter, which leads to diffuse axonal injury $(1,11)$, a characteristic feature of mTBI. Using magnetic resonance imaging (MRI) in conjunction with diffusion tensor imaging (DTI), Davenport et al. (18) demonstrated that a single season of American high school football resulted in changes in MRI indicative of axonal injury in the absence of a concussion diagnosis. This was the first study to demonstrate that the sub-concussive impacts sustained by American football athletes likely result in a measurable physiological response.

Interestingly, elevations in cerebrospinal fluid (CSF) neurofilament light polypeptide (NFL) have been reported in boxers sustaining concussive or sub-concussive head impacts $(32,33,49)$. Neurofilaments are abundant in key intermediate fibers in neurons and major components of the axonal skeleton $(21,38)$ thus, marked elevations indicate a level of axonal injury. While boxers deliver punches with high impact velocity, a lower head injury criterion due to shorter duration acceleration and translational acceleration has been reported when compared to American football (46). Thus, it is likely that the sub-concussive impacts sustained by American football athletes also result in a measurable change in NFL. Indeed, using single molecule array (Simoa), which allows quantification with an increase in analytical sensitivity over that of conventional assays(39), we found that a season of American football results in measurable elevations in serum NFL coincident with those times in which an increase in the number of head impacts was likely (36). This is of great importance, given that in the absence of reporting or characteristic display of symptoms an athlete may play before full recovery, which may further subject the athlete to injury and increase the likelihood of damage to the brain (41).

In their recent position statement, the American Medical Society for Sports Medicine suggested that despite advancements in protective equipment (47), those changes have not been shown to reduce the incidence and/or severity of concussion in sport (23). Our recent findings (36) and those of Davenport et al. (18) highlight the need for a different approach. Nutritional supplementation, specifically docosahexaenoic acid (DHA, 22:6n-3), the principle n-3 long-chain polyunsaturated fatty acid ( $n-3$ LCPUFA) in brain tissue (25) has received considerable attention as a possible intervention to mitigate the pathology associated with $\mathrm{mTBI}$. Prior investigation of traumatic brain injury (TBI) in animal models has demonstrated that supplemental DHA attenuates axonal damage when administered prior to insult $(6,43)$. The typical American diet is scarce in both DHA and the $n-3$ LCPUFA eicosapentaenoic acid (EPA, 20:5n-3), with an estimated combined intake of about $100 \mathrm{mg}^{\circ}$ day $^{-1}$ (20). While it is known that supplemental DHA results in an increase in plasma DHA in a dose dependent manner up to approximately $2 \mathrm{~g} \mathrm{day}^{-1}$ in the average population (5), American football athletes are larger than the average and most athletes (48) and participate in heavy physical training, which is known to affect fatty acid composition (35). Therefore, larger doses may be necessary.

This study sought to examine the effect of differing doses of supplemental DHA on serum NFL over the course of a season of American football. We have previously reported that American football results in measurable elevations in serum NFL over the course of the season (36). We hypothesized that American football athletes would require higher doses of supplemental DHA due to their larger size and physical activity. Further, given reports of supplemental DHA reducing axonal injury in rodent models of TBI $(6,43)$, we hypothesized that supplemental DHA would attenuate the increase in serum NFL observed.

Methods 
Subjects. This study was conducted according to the Declaration of Helsinki guidelines. All procedures involving human subjects were approved by the Institutional Review Board of Texas Christian University for use of human subjects in research (protocol no. 1404-68-1404). Written consent was obtained from all subjects.

All athlete volunteers were cleared to participate in university athletics as determined by the team physician. Exclusion criteria included chronic anti-inflammatory or anti-hypertensive medication use, medications for blood lipids, fish oil or omega-3 fatty acid supplementation, and consumption of more than 2 servings of fish per week. Athletes injured or unable to participate in regularly schedule conditioning or competitions were also excluded. A consort diagram is provided in Figure 1 outlining reasons for dropout and/or exclusion. A total of 81 athletes completed the study.

Insert Figure 1 here

Study Design. A randomized, double-blind, placebo-controlled, parallel design study was employed to examine the effect of differing doses of DHA on plasma fatty acids and serum NFL over the course of a season (189 days) of American football to include off-season summer conditioning (57 days), pre-season camp (23 days), and the competitive season (109 days). Prior to the start of summer conditioning, the

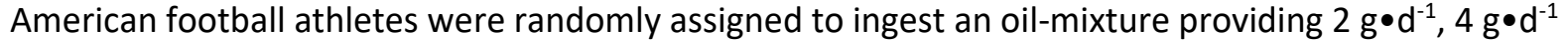
$\mathrm{DHA}, 6 \mathrm{~g} \bullet \mathrm{d}^{-1} \mathrm{DHA}$, or placebo, corn oil, for the study duration. Blood was sampled at specific intervals over the course of the 189 day intervention coincident with changes in intensity, hours of contact, and likely changes in head impacts. Upon reporting for summer conditioning, a baseline blood sample was taken when volunteers returned from a period of no contact ( 9 weeks) (Baseline; T1). Summer conditioning consisted of strength and conditioning workouts Monday through Friday for approximately two hours per day. A second sample was obtained the conclusion of summer training prior to preseason training camp (Pre-Camp; T2). In the time between baseline and the start of training camp no head to head contact took place, but many of the athletes elected to participate in non-supervised seven on seven practices, which involve no contact or equipment and occurred approximately twice per week for one-hour. A third sample was collected following pre-season training camp (Post-Camp; T3). Camp began with three non-contact practices in shorts and helmets, one practice in helmet and shoulder pads, followed by full gear practices. The first five days, practices were held once per day. Thereafter, two-a-day practices were held every other day for the remainder of camp. One day a week (Sunday) the athletes were off from training and practice. The remaining blood samples were taken throughout the competitive season on the Monday following a Saturday game (48 hours) (T4-T8). The first two samplings $(\mathrm{T4}, \mathrm{T5})$ occurred during Pre-conference play, whereas the last three (T6-T8) occurred during conference play. During the competitive season athletes underwent a one hour shorts and helmets practice on Sunday, Monday was an off day, followed by full pad practice on Tuesday, helmets and shoulder pads on Wednesday, Thursday and Friday were shorts and helmets only (no full contact, incidental only). During the competition season, tackles were not taken to the ground, hit and wrap only. Additionally, athletes would perform three strength and conditioning sessions per week. The maximum (median) number of head impacts per season for an NCAA American football athlete has been reported to range from 15.6 to 24 (4.8 to 7.5) and 58.5 to 86.1 (12.1 to 16.3) for practices and games, respectively (14). All samplings throughout the season occurred within 14 - 28 day intervals, with no sampling occurring more than 28 days from the previous one.

Supplementation. Prior to the beginning of summer training, a one-hundred thirty man roster of prospective volunteers from a National Collegiate Athletic Association (NCAA) Division I American football team was provided to research personnel. Potential participants were randomized by player position provided by coaching staff and subsequently randomized to one of four treatment groups ( 2 $\mathrm{g} \bullet \mathrm{d}^{-1} \mathrm{DHA}, 4 \mathrm{~g} \bullet \mathrm{d}^{-1} \mathrm{DHA}, 6 \mathrm{~g} \bullet \mathrm{d}^{-1} \mathrm{DHA}$, or placebo) using a random number generator. The algal DHA used in this study (DHA-S oil, DSM Nutritional Products; Columbia, MD) was derived from Schizochytrium sp. containing $35-45 \%$ DHA by weight. Wesson ${ }^{\circledR}$ corn oil, containing $<0.1 \%$ DHA, was used as the placebo 
and in the $2 \mathrm{~g} \bullet \mathrm{d}^{-1}$ and $4 \mathrm{~g} \bullet \mathrm{d}^{-1} \mathrm{DHA}$ oil-mixtures to ensure equal volume. To ensure proper dose the lower limit (35\%) of DHA content by weight contained in the DHA-S oil was used for calculations. The oil mixture was weighed to the nearest $0.1 \mathrm{~g}$ on food scales (Ohaus CS-200; Parsippany, NJ, USA). All mixtures were flavored with artificial flavoring as a masking agent. Stability of oil mixture was verified by procedures outlined in "Fatty acid composition of plasma" for preparation up to 7 days prior to delivery. The fatty acid composition for both oils is shown in Table 1.

\section{Insert Table 1 here}

Participants were advised as to which foods were high in DHA and asked to limit servings to no more than 2 per week during the study. Supplement adherence was monitored daily via visual supervision by the same research personnel. To ensure compliance, supplement was given only on days when the participants reported to the training facility resulting in participants receiving supplement for 5 days, 7 days, and 5 days per week for summer training, pre-season camp, and the competitive season, respectively. Supplement compliance was set at $\geq 80 \%$ for each individual over the course of the study. Days that supplement was not given were not taken into account for calculating compliance.

Blood sampling and preparation. The night prior to each blood collection, participants were verbally reminded to ingest only water after 2200. On the day of blood sampling, participants reported to the athletic training facility after an overnight fast ( $\geq$ eight hours), supine blood samples were collected via venipuncture from the antecubital fossa region using standard, sterile phlebotomy procedures. To prevent any substantial clinical differences in subsequent blood draws, short-term stasis was prevented by immediate removal of the tourniquet following observable blood flow (27). Blood samples were collected in spray-coated $\mathrm{K}_{2}$ ethylenediaminetetraacetic acid (EDTA) vacutainer tubes and serum vacutainer tube with no additive (BD Diagnostics, Franklin Lakes, NJ, USA). Vacutainer tubes were kept on ice prior to blood collection and immediately placed on ice following blood collection. All samples were centrifuged at 2,000 g for 30 minutes at $4^{\circ}$ Celsius (Beckman Coulter, Allegra X-15R, Brea, CA, USA) within 30 minutes of collection. Aliquots of serum, plasma, and red blood cells, collected via sterile pipette from center of the red blood cell pack within the EDTA vacutainer tubes, were immediately transferred to a pre-labeled polypropylene vials .

Serum Neurofilament Light (NFL). Serum NFL levels were determined using the NF-Light kit from UmanDiagnostics (UmanDiagnostics, Umeå, Sweden), transferred onto the Simoa platform using a homebrew kit (Quanterix Corp, Boston, MA, USA). The lower limit of quantification was $1.95 \mathrm{pg} / \mathrm{mL}$. The analyses were performed by a board-certified laboratory technician in one round of experiments using one batch of reagents with intra-assay coefficients of variation below $10 \%$.

Fatty acid composition of plasma. Total lipid was extracted and methylated according to previously described procedures $(2,22,31)$. Fatty acid methyl esters (FAME) were analyzed with a Varian gas chromatograph (model CP-3800 fixed with a CP-8200 autosampler, Varian Inc.; Walnut Creek, CA) equipped with a fused silica capillary column CP-Sil88 (100 m x 0.25 mm i.d.; Chrompack Inc., Middleburg, The Netherlands) (22). Individual FAME were identified using genuine standards (Nu-Chek Prep, Inc., Elysian, MN) and response factors were calculated from a library of commercially available methyl esters of palmitic, oleic, linoleic, and arachidonic acid from the same manufacturer. Data are expressed as percent (\%) total fatty acids.

Statistical Analyses. A priori power analysis was conducted using G*Power version 3.1.9 to determine the minimum sample size required to find significance in changes in proportion DHA in total plasma fatty acids with a desired level of power set at .80, an $\alpha$-level at .05, and a standardized effect size calculated from previous pilot study. It was determined that total of 20 subjects were needed to ensure adequate power.

The effects of supplementation and time on variables of interest were estimated from a mixed model analysis of variance (ANOVA) in SPSS V.22 (IBM Corporation; Armonk, NY). From that model, estimates and uncertainty of the large-sample effect size for the effect of treatment and time on 
dependent measures were derived from the model to allow a magnitude-based approach to inference (24). Effect sizes were calculated and a modified classification system (trivial, $0.0-0.2$; small, 0.2 - 0.6; moderate, 0.6 - 1.2; large, 1.2-2.0; very large, >2.0; extremely large, >4.0) was used to further interpret the magnitude of change. The probability (likelihood) that a contrast was at least greater than the smallest threshold, calculated as the standardized change of 0.2 times the between subject standard deviation at baseline among all treatments was qualified as follows: $0.5 \%$, almost certainly not; $0.5-5 \%$, very unlikely; 5-25\%, unlikely; $25-75 \%$, possible; $75-95 \%$, likely; $95-99.5 \%$, very likely; $99.5 \%$, almost certain. In the case where the majority ( $>50 \%)$ of the confidence interval $(\mathrm{Cl})$ lies between the threshold for substantiveness and the probability of benefit or harm is $<5.0 \%$, the effect was qualified trivial with the appropriate likelihood qualifier.

\section{Results}

Compliance and Reported Side Effects. Mean compliance over the course of the 189 day intervention

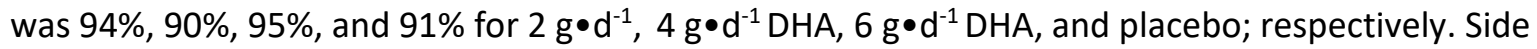
effects of supplemental DHA included initial gastrointestinal distress, poor palatability, and belching. Supplemental DHA Increases Plasma DHA in Dose-Dependent Manner. The change from T1 to T8 in proportion DHA, EPA, and arachidonic acid (ARA) of total plasma fatty acids is shown in Figure 2A-C, respectively. DHA supplementation increased proportion of DHA in plasma fatty acids from baseline by a most likely large magnitude ( $E S=1.74$ to 2.17 ) in a dose-dependent manner. Clear contrasts were observed when examining change from baseline in each dose, with very likely and most likely substantially greater delta in the $6 \mathrm{~g} \bullet \mathrm{d}^{-1} \mathrm{DHA}$ treatment group, when compared to $4 \mathrm{~g} \bullet \mathrm{d}^{-1}(E S=0.7 ; \mathrm{p}=$

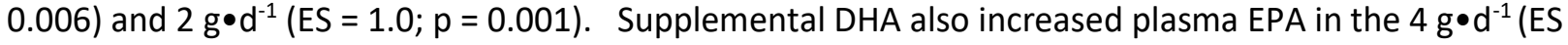
$=0.6 ; p=0.130)$ and $6{\mathrm{~g} \bullet \mathrm{d}^{-1}}^{-1} \mathrm{ES}=1.7 ; \mathrm{p}<0.001)$ treatment groups. However, clear contrasts were only observed in the $6 \mathrm{~g} \mathrm{~d}^{-1}$ dose (mean difference; $\pm 90 \% \mathrm{Cl} ; 0.23 ; \pm 0.15 \%$ ) when change from baseline was compared to placebo $(E S=0.9 ; p=0.016)$. ARA decrease in all DHA treatment groups, with the most

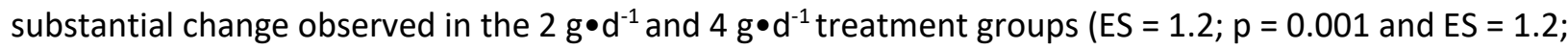
$p=0.002$, respectively).

Insert Figure 2 here

Serum Neurofilament Light (NFL) Increases in Starters. Serum NFL increased substantially over the course of the season in those participants categorized as starters (Figure 3A). The observed increase occurred coincident with changes in intensity and hours of contact. The Post-Camp (T3) serum NFL increase observed was very likely, but small in magnitude in starters ( $E S=0.4 ; p=0.001$ ) compared to baseline (T1). As expected, a similar albeit smaller increase was observed in non-starter (ES $=0.3 ; p=$ 0.043 ) as those in this category would have experienced the most contact during the time from PreCamp (T2) to Post-Camp (T3). All other contrasts in the non-starters were trivial. However, serum NFL remained elevated the remainder of the competition season in starters, with a further very likely increase of moderate magnitude occurring at T6 (ES $=0.6 ; p<0.001)$, coincident with conference play. A further increase was noted at T7 (ES $=0.8 ; p<0.001)$, which remained elevated to the end of the competitive season ( $E S=0.7 ; p<0.001)$. The increase observed over the course of the study in starters resulted in substantial differences noted over the course of the study when compared to non-starters (Table 3). Similarly, the area under the curve calculation determination resulted in a very likely difference of moderate magnitude $\left(600 ; \pm 430 \mathrm{pg} \bullet \mathrm{mL}^{-1} \bullet\right.$ day; $\left.E S=0.6 ; \mathrm{p}=0.024\right)$ between starters (1995 $\pm 1383 \mathrm{pg} \bullet \mathrm{mL}^{-1} \bullet$ day $)$ and non-starters $\left(1398 \pm 581 \mathrm{pg} \bullet \mathrm{mL}^{-1} \bullet\right.$ day $)$.

Insert Figure 3 here

Insert Table 2 here 
Supplemental DHA Attenuates Increase in Serum NFL. Due to the lack of change in serum NFL, those athletes categorized as non-starters were excluded from further analyses. The effect of DHA supplementation irrespective of dose (collapsed across treatments) compared to placebo is presented in Figure 3B whereas Figure $3 \mathrm{C}$ shows the effect of the different doses on serum NFL over the course of football season. When collapsed across all treatment groups, supplemental DHA likely attenuated serum NFL Post-Camp ( $p=0.070$; Table 3), coincident with Post-Camp (T3) in which a substantial increase in serum NFL was observed in starters. During conference play, when a second substantial increase in serum NFL was observed amongst starters, supplementation with DHA resulted in a likely lowering effect compared to placebo $(p=0.144, T 6)$, which continued through the end of the study when a likely lowering of serum NFL was observed in those supplementing with DHA compared to placebo $(p=0.022, T 7 ; p=$ $0.012, T 8)$. Examination of the effect of the differing doses on serum NFL resulted in fewer athletes in each group. However, we observed a likely and very likely substantial lowering effect of $2{\mathrm{~g} \bullet \mathrm{d}^{-1} \mathrm{DHA}}^{-1}$

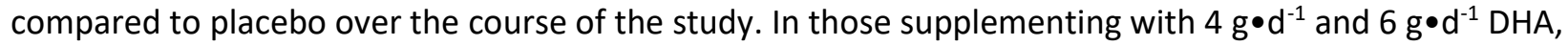
all contrasts were unclear with the exception of the final time point in which a likely substantial lowering

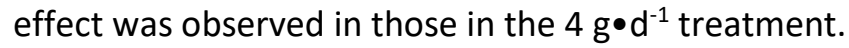

Insert Table 3 here

\section{Discussion}

In this study, we examined the effect of differing doses of DHA on plasma fatty acids and serum NFL, a biomarker of head trauma, over the course of a season in American football athletes. This study provides novel data specific to the optimal dose of DHA in American football athletes. Further, the data presented herein demonstrate that a season of American football is associated with some level of subconcussive injury, which results in a measurable increase in a marker of axonal damage. Most importantly, we report for the first time that supplemental DHA, irrespective of dose, likely attenuates elevations in serum NFL coincident with those times in which an increased number and magnitude of head impacts is likely in American football athletes.

The neuroprotective effects of supplemental DHA observed in rodent models of TBI are most evident when doses are high ( $40 \mathrm{mg} \bullet \mathrm{kg}^{-1} \bullet$ day) (7), corresponding to a dose of approximately $4.3 \mathrm{~g} \bullet \mathrm{kg}$ ${ }^{1} \cdot$ day in today's collegiate American football athlete $(28,37)$. However, in analyzing the dose-response effect, Arterburn et al. (5) reported a dose-dependent relationship in which plasma DHA increases up to a dosage of approximately $2 \mathrm{~g} \bullet \mathrm{day}^{-1}$; above this dose, the authors reported only incremental increases are observed as saturation is approached (5). The finding of a dose-response effect is in agreement

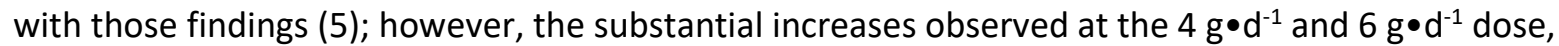
suggests that higher doses may be necessary as no apparent plateau was noted. This is not surprising given the large size of American football athletes compared to the general population and other athletes (48). Despite having relatively high body mass, the subjects in those studies analyzed by Arterburn et al. (2) were lower than those reported in American football athletes $(28,37)$. Further, American football athletes undergo rigorous physical training physical activity, which is known to influence plasma fatty acid composition (35).

The lack of an observable increase in EPA in those receiving $2 \mathrm{~g} \bullet \mathrm{d}^{-1}$ combined with a lack of clear contrasts when comparing change from baseline in those receiving $4{\mathrm{~g} \bullet \mathrm{d}^{-1}}^{1}$ and placebo supports the assertion that a higher dose may be necessary in American football athletes. While retroconversion of DHA to EPA is regularly observed $(5,30)$, Mori et al. (29) reported a small non-significant increase in EPA following a 6-week intervention in which overweight men received supplemental DHA in a dose of $4 \mathrm{~g} \bullet \mathrm{d}$ ${ }^{1}$. Interestingly, the largest decrease in ARA occurred in those receiving $2 \mathrm{~g} \bullet^{-1}$. Though a similar dose dependent reduction in ARA is typically observed, the response has been reported to be variable when 
examining a number of studies (5). This study contributes important new information specific to optimizing DHA supplementation in American football athletes.

It is well known that American football athletes are exposed to head impacts that vary in number and magnitude over the course of a season (14). Further, those routine head impacts sustained result in rotational acceleration/deceleration and propagation of force through the brain $(9,15,40)$, impacts known to cause diffuse axonal injury $(1,11)$. It has been suggested that NFL, albeit in CSF, is one of the most sensitive biomarkers of head trauma, specifically axonal injury $(32,49,50)$. Recently, Al Nimer et al. (3) reported that a significant relationship exists between CSF NFL and serum NFL in severe TBI. As such, elevations of NFL in peripheral blood would indicate some level of axonal injury. Thus, the finding of marked elevations following periods in which an increase in number and magnitude of head impacts was likely (14) suggests that some level of axonal damage occurs in American football athletes over the course of a season in the absence of a concussion diagnosis.

Indeed, Davenport et al. (18) reported that the risk weighted exposure (RWE), a metric defined as collected risk of concussion over the course of a season(45), showed strong associations with diffusion tensor imaging (DTI) scalars in American football athletes at the high school level in the absence of a concussion diagnosis. Most studies using DTI in TBI and mTBI report that the changes reported in those specific scalars identified by Davenport et al. (18) result from axonal injury $(4,34)$. The number of high-level impacts are more frequent in collegiate compared to high school players (42); thus, our data support that the sub-concussive impacts sustained by collegiate American football players results in some degree of axonal injury. The primary inferential limitation to the current data is the lack of an outcome measure related to the actual number of head impacts sustained over the course of the season. As a result, our ability to discern if the elevations in serum NFL were a result of axonal damage caused by head impacts or from another source, such as muscle is limited. However, despite participating in practice over the course of the season, serum NFL did not vary in those categorized as non-starters, suggesting that the elevations observed herein were in fact a result of impacts to the head.

Perhaps the most novel finding of the current study was that supplemental DHA likely

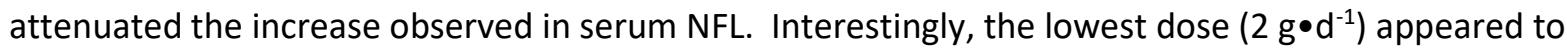
produce the most marked reductions in serum NFL compared to placebo. However, inference about the differing doses is limited by the number of starters in each group. Previous studies in rodent models of TBI support the possibility that DHA reduces markers of axonal injury $(6,43)$. Fewer numbers of $\beta$ amyloid precursor protein (APP)- positive axons, a marker of axonal injury, were reported in animals receiving supplemental DHA for 30 days prior to impact acceleration injury (6). More recently, Schober et al. (43) examined the effect of DHA on DTI indices of white matter injury using an established model for pediatric TBI, controlled cortical impact (CCI) (26). DHA decreased the increment in radial diffusivity (RD) observed in the DTI following CCl, increased RD is correlated with histologic axonal damage(44). Thus, our data are in support of previous studies in which DHA has been shown to provide neuroprotective effect in rodent models of TBI. While a number of mechanisms have been proposed, the exact mechanism underlying the neuroprotective effects of DHA are as of yet unknown. Our findings are in support of those findings in which DHA attenuated markers of axonal injury in rodent models. However, we report on the potential effectiveness of DHA for axonal injury caused by sub-concussive impacts in American football athletes, the first to date.

The current study has several limitations. First, our results are limited by the sample size, particularly as it relates to starters. Based on preliminary data and the absence of an optimal dose regimen for DHA for the larger American football athlete at the time of design, we sought to experimentally define an optimal dosage. In doing so, this limited the number of starters in each dose treatment group making interpretation of the optimal dose for neuroprotective effect of DHA difficult. Still, as mentioned in the Results, when collapsed across treatment the likely lowering effect of DHA on

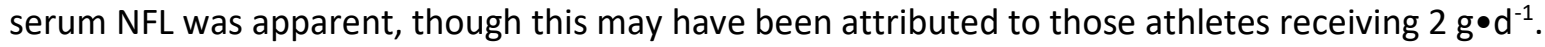


Second, the lack of an outcome measure for number of head impacts limits inference as to the origination of NFL appearing in peripheral blood. No increase was observed in the non-starters participating in strenuous practice which suggests that NFL was indeed from head impacts. Further study is warranted to examine the relationship between the number of head impacts and serum NFL levels in this population. The inclusion of DTI would provide additional support.

In conclusion, the data presented herein demonstrate that American football athletes likely require a higher dose of DHA than the average population. Further, serum NFL increases in those categorized as starters coincident with those times in which the number of head impacts likely increases. Most importantly, we report for the first time that supplemental DHA likely attenuates the increase in serum NFL suggesting a neuroprotective effect of DHA, specifically as it relates to axonal injury. The latter agrees with previous reports of the neuroprotective effect of DHA in rodent models of TBI. Our study contributes important information on the effects of DHA on biological markers of head trauma in a population known to sustain a significant number of head impacts. Given the potential of DHA to provide neuroprotection and the fact that it is well-tolerated and safe at any age, further study is warranted to elucidate the true nature of the effect.

\section{Reference List}

1. Adams JH, Doyle D, Ford I, Gennarelli T, Graham D, McLellan D. Diffuse axonal injury in head injury: definition, diagnosis and grading. Histopathology. 1989;15(1):49-59.

2. Adams TH, Walzem RL, Smith DR, Tseng S, Smith SB. Hamburger high in total saturatred and trans-fatty acids decreases HDL cholesterol and LDL particle diameter, increases plasma TAG, in mildly hypercholesterolaemic men. Br J Nutr. 2010;103:91-8.

3. Al Nimer F, Thelin E, Nyström $\mathrm{H}$ et al. Comparative Assessment of the Prognostic Value of Biomarkers in Traumatic Brain Injury Reveals an Independent Role for Serum Levels of Neurofilament Light. PLOS ONE. 2015;10(7):e0132177.

4. Arfanakis K, Haughton VM, Carew JD, Rogers BP, Dempsey RJ, Meyerand ME. Diffusion tensor MR imaging in diffuse axonal injury. Am J Neuroradiol. 2002;23(5):794-802.

5. Arterburn LM, Bailey Hall E, Oken H. Distribution, interconversion, and dose response of $n-3$ fatty acids in humans. Am J Clin Nutr. 2006;83(suppl):1467S-76S.

6. Bailes JE, Mills JD. Docosahexaenoic acid reduces traumatic axonal injury in a rodent head injury model. J Neurotrauma. 2010;27:1617-24.

7. Bailes JE, Patel V. The potential for DHA to mitigate mild traumatic brain injury. Mil Med. 2014;179(11S):112-6.

8. Bermunt SH, Mensink rP, Simonis MM, Hornstra G. Effects of dietary alpha-linolenic acid on the conversion and oxidation of ${ }^{13} \mathrm{C}$-alpha-linolenic acid. Lipids. 2000;35:137-42.

9. Broglio SP, Schnebel B, Sosnoff JJ et al. The biomechanical properties of concussions in high school football. Med Sci Sports Exerc. 2010;42(11):2064.

10. Broglio SP, Sosnoff JJ, Shin S, He X, Alcaraz C, Zimmerman J. Head impacts during high school football: a biomechanical assessment. J Athl Train. 2009;44(4):342.

11. Browne KD, Chen X-H, Meaney DF, Smith DH. Mild traumatic brain injury and diffuse axonal injury in swine. J Neurotrauma. 2011;28(9):1747-55.

12. Burdge GC, Finnegan YE, Minihane AM, Williams CM, Wootton SA. Effect of altered n-3 fatty acid intake upon plasma lipid fatty acid composition, conversion of $\left[{ }^{13} \mathrm{C}\right]$ alpha linolenic acid to longer-chaing fatty acids and partitioning towards beta-oxidation in older men. Br J Nutr. 2003;90:311-21.

13. Burdge GC, Jones AE, Wootton SA. Eicosapentaenoic and docosapentaenoic acids are the principal prducts of alpha linolenic acid metabolism in young men. Br J Nutr. 2002;88:355-64. 
14. Crisco JJ, Fiore R, Beckwith JG et al. Frequency and location of head impact exposures in individual collegiate football players. J Athl Train. 2010;45(6):549.

15. Crisco JJ, Wilcox BJ, Beckwith JG et al. Head impact exposure in collegiate football players. J Biomech. 2011;44(15):2673-8.

16. Daneshvar DH, Nowinski CJ, McKee A, Cantu RC. The epidemiology of sport-related concussion. Clin Sports Med. 2011;30(1):1-17.

17. Daniel RW, Rowson S, Duma SM. Head impact exposure in youth football. Ann Biomed Eng. 2012;40(4):976-81.

18. Davenport EM, Whitlow CT, Urban JE et al. Abnormal white matter integrity related to head impact exposure in a season of high school varsity football. J Neurotrauma. 2014;31(19):1617-

24.

19. DeKosky ST, Blennow K, Ikonomovic MD, Gandy S. Acute and chronic traumatic encephalopathies: pathogenesis and biomarkers. Nat Rev Neurol. 2013;9(4):192-200.

20. Ervin RWJ, Wang C, Kennedy-Stephenson J. Dietary intake of fats and fatty acids for the United States population: 1999-2000. Adv Data Vital Health Stat. 2004;348:1-6.

21. Fletcher DA, Mullins RD. Cell mechanics and the cytoskeleton. Nature. 2010;463(7280):485-92.

22. Folch J, Lees M, Sloane Stanley GH. A simple method for the isolation and purification of total lipids from animal tissues. J Biol Chem. 1957;226(1):497-509.

23. Harmon KG, Drezner JA, Gammons M et al. American Medical Society for Sports Medicine position statement: Concussion in sport. Br J Sports Med. 2013;47(1):15-26.

24. Hopkins W, Marshall S, Batterham A, Hanin J. Progressive statistics for studies in sports medicine and exercise science. Med Sci Sports Exerc. 2009;41(1):3.

25. Kim HY. Novel metabolism of docosahexaenoic acid in neural cell. J Biol Chem. 2007;26:18661-5.

26. Lighthall JW. Controlled cortical impact: a new experimental brain injury model. J Neurotrauma. 1988;5(1):1-15.

27. Lippi G, Salvagno GL, Montagnana M, Brocco G, Guidi GC. Influence of short-term venous stasis on clinical chemistry testing. Clin Chem Lab Med. 2005;43(8):869-75.

28. Melvin MN, Smith-Ryan AE, Wingfield HL, Ryan ED, Trexler ET, Roelofs EJ. Muscle characteristics and body Composition of NCAA division I football players. J Strength Cond Res. 2014;28(12):3320-9.

29. Mori TA, Bao DQ, Burke V, Puddey IB, Beilin LJ. Docosahexaenoic acid but not eicosapentaenoic acid lowers ambulatory blood pressure and heart rate in humans. Hypertension. 1999;34(2):25360.

30. Mori TA, Burke V, Puddey IB et al. Purified eicosapentaenoic and docosahexaenoic acids have differential effects on serum lipids and lipoproteins, LDL particle size, glucose, and insulin in mildly hyperlipidemic men. Am J Clin Nutr. 2000;71(5):1085-94.

31. Morrison W, Smith L. Preparation of fatty acid methyl esters and dimethylacetals from lipics with boron fluoride-methanol. J Lipid Res. 1964;5:600-8.

32. Neselius S, Brisby H, Marcusson J, Zetterberg H, Blennow K, Karlsson T. Neurological assessment and its relationship to CSF biomarkers in amateur boxers. PLOS ONE. 2014;9(6):e99870.

33. Neselius S, Brisby H, Theodorsson A, Blennow K, Zetterberg H, Marcusson J. CSF-biomarkers in Olympic boxing: diagnosis and effects of repetitive head trauma. PLOS ONE. 2012;7(4):e33606.

34. Newcombe V, Williams $G$, Nortje J et al. Analysis of acute traumatic axonal injury using diffusion tensor imaging. Br J Neurosurg. 2007;21(4):340-8.

35. Nikolaidis MG, Mougios V. Effects of exercise on the fatty-acid composition of blood and tissue lipids. Sports Med. 2004;34(15):1051-76.

36. Oliver JM, Jones MT, Kirk KM et al. Serum neurofilament light in American football athletes over the course of a season. Journal of Neurotrauma. In review. 
37. Oliver JM, Lambert BS, Martin SE, Green JS, Crouse SF. Predicting football players' dual-energy xray absorptiometry body composition using standard anthropometric measures. J Athl Train. 2012;47(3):257.

38. Perrot R, Berges R, Bocquet A, Eyer J. Review of the multiple aspects of neurofilament functions, and their possible contribution to neurodegeneration. Mol Neurobiol. 2008;38(1):27-65.

39. Rissin DM, Kan CW, Campbell TG et al. Single-molecule enzyme-linked immunosorbent assay detects serum proteins at subfemtomolar concentrations. Nature Biotechnol. 2010;28(6):595-9.

40. Rowson S, Duma SM, Beckwith JG et al. Rotational head kinematics in football impacts: an injury risk function for concussion. Ann Biomed Eng. 2012;40(1):1-13.

41. Saunders RL, Harbaugh RE. The second impact in catastrophic contact-sports head trauma. JAMA. 1984;252(4):538-9.

42. Schnebel B, Gwin JT, Anderson S, Gatlin R. In vivo study of head impacts in football: A comparison of National Collegiate Athletic Association Division I versus high school impacts. Neurosurgery. 2007;60(3):490-6.

43. Schober ME, Requena DF, Abdullah OM et al. Dietary docosahexaenoic acid Improves cognitive function, tissue sparing, and magnetic resonance imaging indices of edema and white matter injury in the immature rat after traumatic brain injury. J Neurotrauma. 2015.

44. Tuor UI, Morgunov M, Sule M et al. Cellular correlates of longitudinal diffusion tensor imaging of axonal degeneration following hypoxic-ischemic cerebral infarction in neonatal rats. Neurolmage: Clinical. 2014;6:32-42.

45. Urban JE, Davenport EM, Golman AJ et al. Head impact exposure in youth football: high school ages 14 to 18 years and cumulative impact analysis. Ann Biomed Eng. 2013;41(12):2474-87.

46. Viano DC, Casson IR, Pellman EJ et al. Concussion in professional football: Comparison with boxing head impacts-Part 10. Neurosurgery. 2005;57(6):1154-72.

47. Viano DC, Halstead D. Change in size and impact performance of football helmets from the 1970s to 2010. Ann Biomed Eng. 2012;40(1):175-84.

48. Yamamoto JB, Yamamoto BE, Yamamoto PP, Yamamoto LG. Epidemiology of college athlete sizes, 1950s to current. Res Sports Med. 2008;16:111-27.

49. Zetterberg $\mathrm{H}$, Hietala MA, Jonsson $\mathrm{M}$ et al. Neurochemical aftermath of amateur boxing. Arch Neurol. 2006;63(9):1277-80.

50. Zetterberg $\mathrm{H}$, Smith $\mathrm{DH}$, Blennow K. Biomarkers of mild traumatic brain injury in cerebrospinal fluid and blood. Nat Rev Neurol. 2013;9(4):201-10. 
Figure Legends.

Figure 1. Consort diagram outlining reasons for exclusions. DHA, docosahexaenoic acid.

Figure 2. Effect of docosahexaenoic acid (DHA) supplementation on proportion arachidonic acid (A), eicosapentaenoic acid (EPA) (B), and DHA of total plasma fatty acids in all treatment groups.; Threshold for smallest substantial change calculated as 0.2 times baseline $\mathrm{SD}_{\text {between; }}$ Likelihood is shown as increased number of symbols (+)relative to baseline in each treatment group: +possible, ++ likely, +++ very likely, ++++ most likely. All data are mean. A magnitude-based summary of statistical contrasts among treatments with corresponding mean \pm SD is shown in Table 2.

Figure 3. (A) Changes in serum neurofilament light $\left(\mathrm{pg} \bullet \mathrm{mL}^{-1}\right)$ over the course of the study in starters and non-starters; (B \& C) Effect of supplemental docosahexaenoic acid (DHA) on serum neurofilament light (\% change from baseline) over the course of the study in starters; (B) Collapsed across all doses (A) and (C) separated by dosage; Threshold for smallest substantial change calculated as 0.2 times baseline $\mathrm{SD}_{\text {between; }}$ Likelihood is shown as increased number of symbols (+ used for example) and represented by unique symbol: $(+)$ relative to baseline, $\left({ }^{*}\right)$ relative to placebo. +possible, ++ likely, +++ very likely, ++++ most likely. All data are mean. A magnitude-based summary of statistical contrasts between starters and non-starters with corresponding mean \pm SD is shown in Table 2 and statistical contrasts comparing each dose to placebo with corresponding mean \pm SD is shown in Table 3. 